\title{
ON THE ORDER OF THE ERROR FUNCTION OF THE $k$-FREE INTEGERS
}

\author{
D. SURYANARAYANA AND R. SITARAMACHANDRA RAO
}

ABstract. Let $\Delta_{k}(x)$ and $\Delta_{k}{ }^{\prime}(x)$ be the error functions in the asymptotic formulae for the number and the sum of $k$-free integers $\leqq x$. On the assumption of the Riemann hypothesis, we prove the following results by elementary methods:

$$
\Delta_{k}^{\prime}(x)-x \Delta_{k}(x)=O\left(x^{1+3 !(4 k+1)+\epsilon}\right)
$$

and

where $\epsilon>0$.

$$
\frac{1}{x} \int_{1}^{x} \Delta_{k}(t) d t=O\left(x^{3 /(4 k+1)+\epsilon}\right),
$$

1. Introduction. Let $k$ be a fixed integer $\geqq 2$. A positive integer $n$ is called $k$-free, if $n$ is not divisible by the $k$ th power of any prime. Let $q_{k}(n)$ be the characteristic function of the set of $k$-free integers; that is, $q_{k}(n)=1$ or 0 according as $n$ is $k$-free or not. Let $x$ denote a real variable $\geqq 1,[x]$ denote the greatest integer $\leqq x$ and $\{x\}$ denote the fractional part of $x$; that is, $\{x\}=x-[x]$.

Let $Q_{k}(x)$ and $Q_{\boldsymbol{k}}^{\prime}(x)$ respectively denote the number and the sum of $k$-free integers $\leqq x$. Let

$$
\Delta_{k}(x)=Q_{k}(x)-\frac{x}{\zeta(k)}
$$

and

$$
\Delta_{k}^{\prime}(x)=Q_{k}^{\prime}(x)-\frac{x^{2}}{2 \zeta(k)},
$$

where $\zeta(k)$ is the Riemann zeta function. It is well known that $\Delta_{k}(x)=O\left(x^{1 / k}\right)$. The best known result has been obtained by $\mathrm{A}$. Walfisz [4], viz., $\Delta_{k}(x)=O\left(x^{1 / k} \exp \left(-c_{k} \log ^{3 / 5} x(\log \log x)^{-1 / 5}\right)\right)$, where $c_{k}>0$.

In 1911, A. Axer [1] proved on the assumption of the Riemann hypothesis that $\Delta_{k}(x)=O\left(x^{2 /(2 k+1)+\epsilon}\right)$, for every $\epsilon>0$. The object of the present paper is to establish the following results on the assumption of the Riemann hypothesis:

$$
\Delta_{k}^{\prime}(x)-x \Delta_{k}(x)=O\left(x^{1+3 /(4 k+1)+\varepsilon}\right),
$$

and

Received by the editors April 1, 1970.

AMS 1970 subject classifications. Primary 10H15.

$K e y$ words and phrases. $k$-free intergers, Riemann hypothesis.

Copyright (c) 1971, American Mathematical Society 


$$
\frac{1}{x} \int_{1}^{x} \Delta_{k}(t) d t=O\left(x^{3 /(4 k+1)+e}\right) .
$$

It is reasonable to conjecture that $\Delta_{k}(x)=O\left(x^{3 /(4 k+1)+\epsilon}\right)$.

The method of the paper is elementary and we assume throughout that the Riemann hypothesis is true. We prepare the necessary background in $\S 2$ and give the proofs of (1.3) and (1.4) in $\S 3$.

2. Auxiliary lemmas. In this section we prove some lemmas which are needed in our present discussion. Throughout the following $\epsilon$ denotes any arbitrary positive number.

Lemma 2.1. (Cf. [2, Theorem 14.25(c), p. 315].) If $\mu(n)$ denotes the Möbius function, then

$$
M(x)=\sum_{n \leq x} \mu(n)=O\left(x^{i / 2+\varepsilon}\right)
$$

LEMMA 2.2.

$$
N(x)=\sum_{n>x} \frac{\mu(n)}{n^{k}}=O\left(x^{-k+1 / 2+\epsilon}\right) .
$$

Proof. Putting $f(n)=1 / n^{k}$ it is not hard to show that $f(n+1)$ $-f(n)=O\left(1 / n^{k+1}\right)$. Therefore by partial summation and (2.1),

$$
\begin{aligned}
\sum_{n>x} \mu(n) f(n) & =-M(x) f([x]+1)-\sum_{n>x} M(n)(f(n+1)-f(n)) \\
& =O\left(x^{-k+1 / 2+\epsilon}\right)+O\left(\sum_{n>x} n^{-k-1 / 2+\epsilon}\right) \\
& =O\left(x^{-k+1 / 2+\epsilon}\right)+O\left(x^{-k+1 / 2+\epsilon}\right) .
\end{aligned}
$$

Hence Lemma 2.2 follows.

LEMMA 2.3.

$$
L(x)=\sum_{n \leqq x} \mu(n) n^{k}=O\left(x^{k+1 / 2+\epsilon}\right) .
$$

Proof. Putting $g(n)=n^{k}$ it is clear that $g(n+1)-g(n)=O\left(n^{k-1}\right)$. Therefore by partial summation and (2.1),

$$
\begin{aligned}
\sum_{n \leq x} \mu(n) g(n) & =M(x) g([x])-\sum_{n \leq x-1} M(n)(g(n+1)-g(n)) \\
& =O\left(x^{k+1 / 2+\epsilon}\right)+O\left(\sum_{n \leq x-1} n^{k-1 / 2+\epsilon}\right) \\
& =O\left(x^{k+1 / 2+\epsilon}\right)+O\left(x^{k+1 / 2+\epsilon}\right) .
\end{aligned}
$$


Hence Lemma 2.3 follows.

LemMa 2.4. If $z=(x)^{1 / k}$ and $\rho=\rho(x)$ is any function of $x$ such that $0<\rho<1$, then

$$
\Delta_{k}(x)=-\sum_{n \leq \rho z} \mu(n)\left\{\frac{x}{n^{k}}\right\}+O\left(\rho^{-k+1 / 2+\epsilon} z^{1 / 2+\iota}\right) .
$$

Proof. We have $q_{k}(n)=\sum_{d k \mid n} \mu(d)$, so that

$$
\begin{aligned}
Q_{k}(x) & =\sum_{n \leqq x} q_{k}(n)=\sum_{n \leqq x} \sum_{d^{k} \delta=n} \mu(d)=\sum_{d^{k} \delta \leq x} \mu(d) \\
& =\sum_{d^{k} \delta \leq x ; d \leqq \rho z} \mu(d)+\sum_{d^{k} \delta \leq x ; \delta \leq \rho^{-k}} \mu(d)-\sum_{d \leqq \rho z ; \delta \rho^{-k}} \mu(d) \\
& =S_{1}+S_{2}-S_{3}, \quad \text { say. }
\end{aligned}
$$

Now,

$$
\begin{aligned}
S_{1} & =\sum_{d \leq \rho z} \mu(d) \sum_{\delta \leq x / d k} 1=\sum_{d \leq \rho z} \mu(d)\left[\frac{x}{d^{k}}\right]=x \sum_{d \leq \rho z} \frac{\mu(d)}{d^{k}}-\sum_{d \leq \rho z} \mu(d)\left\{\frac{x}{d^{k}}\right\} \\
& =\frac{x}{\zeta(k)}-x \sum_{n>\rho z} \frac{\mu(n)}{n^{k}}-\sum_{n \leq \rho z} \mu(n)\left\{\frac{x}{n^{k}}\right\} \\
& =\frac{x}{\zeta(k)}-\sum_{n \leq \rho z} \mu(n)\left\{\frac{x}{n^{k}}\right\}+O\left(\rho^{-k+1 / 2+\epsilon} z^{1 / 2+\epsilon}\right),
\end{aligned}
$$

by Lemma 2.2 .

$$
\begin{aligned}
S_{2} & =\sum_{d^{k} \delta \leq x ; \delta \leq \rho^{-k}} \mu(d)=\sum_{\delta \leq \rho^{-k}} \sum_{d \leq(x / \delta)^{1 / k}} \mu(d)=\sum_{\delta \leq \rho^{-k}} M\left(\left(\frac{x}{\delta}\right)^{1 / k}\right) \\
& =O\left(\sum_{\delta \leq \rho^{-k}}\left(\left(\frac{x}{\delta}\right)^{1 / k}\right)^{1 / 2+\epsilon}\right)=O\left(z^{1 / 2+\epsilon} \sum_{\delta \leq \rho^{-k}} \delta^{-1 / 2 k-\epsilon / k}\right) \\
& =O\left(z^{1 / 2+\epsilon}\left(\rho^{-k}\right)^{1-1 / 2 k-\epsilon / k}\right)=O\left(\rho^{-k+1 / 2+\epsilon} z^{1 / 2+\epsilon}\right),
\end{aligned}
$$

by Lemma 2.1 .

$$
S_{3}=\sum_{d \leq \rho ; ; \leq \rho^{-k}} \mu(d)=M(\rho z) \sum_{\delta \leq \rho^{-k}} 1=O\left(\rho^{-k+1 / 2+\epsilon^{1 / 2+e}}\right),
$$

by Lemma 2.1 .

Now, substituting the values of $S_{1}, S_{2}$ and $S_{3}$ in $Q_{k}(x)=S_{1}+S_{2}-S_{3}$, we get Lemma 2.4 in virtue of (1.1).

As a consequence of Lemma 2.4, we get an alternative and an elementary proof of A. Axer's result mentioned in the introduction, viz., 


$$
\Delta_{k}(x)=O\left(x^{2 /(2 k+1)+\epsilon}\right) .
$$

Proof. By (2.4), we have $\Delta_{k}(x)=O(\rho z)+O\left(\rho^{-k+1 / 2+\epsilon} z^{1 / 2+\epsilon}\right)$. Now, taking $\rho=x^{-1 / k(2 k+1)}$, we see that the first $O$-term is $O\left(x^{2 /(2 k+1)}\right)$ and the second $O$-term is $O\left(x^{2 /(2 k+1)+2 \epsilon /(2 k+1)}\right)$. Hence (2.5) follows.

LEMMA 2.5. If $z=(x)^{1 / k}$ and $\rho=\rho(x)$ is any function of $x$ such that $0<\rho<1$, then

$$
\Delta_{k}^{\prime}(x)=-x \sum_{n \leq \rho z} \mu(n)\left\{\frac{x}{n^{k}}\right\}+O\left(\rho^{k+1} z^{k+1}\right)+O\left(\rho^{-k+1 / 2+\epsilon} z^{k+1 / 2+\epsilon}\right)
$$

Proof. We have

$$
\begin{aligned}
Q_{k}^{\prime}(x) & =\sum_{n \leqq x} Q_{k}(n) n=\sum_{n \leqq x} n \sum_{d^{k} \delta=n} \mu(d)=\sum_{d^{k} \delta \leqq x} \mu(d) d^{k} \delta \\
& =\sum_{d^{k} \delta \leqq x ; d \leqq \rho z} \mu(d) d^{k} \delta+\sum_{d^{k} \delta \leqq x ; \delta \leqq \rho^{-k}} \mu(d) d^{k} \delta-\sum_{d \leqq \rho z \delta \leq \rho^{-k}} \mu(d) d^{k} \delta \\
& =S_{1}^{\prime}+S_{2}^{\prime}-S_{3}^{\prime}, \quad \text { say. }
\end{aligned}
$$

Now,

$$
\begin{aligned}
& S_{1}^{\prime}=\sum_{d \leq \rho z} \mu(d) d^{k} \sum_{\delta \leq x / d^{k}} \delta=\sum_{d \leq \rho z} \mu(d) d^{k} \frac{1}{2}\left(\left[\frac{x}{d^{k}}\right]^{2}+\left[\frac{x}{d^{k}}\right]\right) \\
& =\frac{1}{2} \sum_{d \leq \rho z} \mu(d) d^{k}\left(\left(\frac{x}{d^{k}}-\left\{\frac{x}{d^{k}}\right\}\right)^{2}+\left(\frac{x}{d^{k}}-\left\{\frac{x}{d^{k}}\right\}\right)\right) \\
& =\frac{1}{2} \sum_{d \leq \rho z} \mu(d) d^{k}\left(\frac{x^{2}}{d^{2 k}}-2 \frac{x}{d^{k}}\left\{\frac{x}{d^{k}}\right\}+\left\{\frac{x}{d^{k}}\right\}^{2}+\frac{x}{d^{k}}-\left\{\frac{x}{d^{k}}\right\}\right) \\
& =\frac{x^{2}}{2} \sum_{d \leq \rho z} \frac{\mu(d)}{d^{k}}-x \sum_{d \leq \rho z} \mu(d)\left\{\frac{x}{d^{k}}\right\}+\frac{1}{2} \sum_{d \leq \rho z} \mu(d) d^{k}\left\{\frac{x}{d^{k}}\right\}^{2} \\
& +\frac{x}{2} \sum_{d \leq \rho z} \mu(d)-\frac{1}{2} \sum_{d \leq \rho z} \mu(d) d^{k}\left\{\frac{x}{d^{k}}\right\} \\
& =\frac{x^{2}}{2 \zeta(k)}-\frac{x^{2}}{2} \sum_{n>\rho z} \frac{\mu(n)}{n^{k}}-x \sum_{n \leq \rho z} \mu(n)\left\{\frac{x}{n^{k}}\right\}+O\left(\rho^{k+1} z^{k+1}\right) \\
& +O\left(\rho^{1 / 2+\epsilon} z^{k+1 / 2+\epsilon}\right)+O\left(\rho^{k+1} z^{k+1}\right) \\
& =\frac{x^{2}}{2 \zeta(k)}-x \sum_{n \leqq \rho z} \mu(n)\left\{\frac{x}{n^{k}}\right\}+O\left(\rho^{-k+1 / 2+\epsilon} z^{k+1 / 2+\epsilon}\right) \\
& +O\left(\rho^{k+1} z^{k+1}\right)+O\left(\rho^{1 / 2+\epsilon} z^{k+1 / 2+\epsilon}\right),
\end{aligned}
$$

by Lemma 2.2. Since $\rho^{k}<1$, the last $O$-term in the above is $O\left(\rho^{-k+1 / 2+\epsilon} z^{k+1 / 2+\epsilon}\right)$, so that 


$$
\begin{aligned}
S_{1}^{\prime} & =\frac{x^{2}}{2 \zeta(k)}-x \sum_{n \leq \rho z} \mu(n)\left\{\frac{x}{n^{k}}\right\}+O\left(\rho^{k+1} z^{k+1}\right)+O\left(\rho^{-k+1 / 2+\epsilon} z^{k+1 / 2+\epsilon}\right) \\
S_{2}^{\prime} & =\sum_{\delta \leqq \rho^{-k}} \delta \sum_{d \leqq(x / \delta)^{1 / k}} \mu(d) d^{k}=\sum_{\delta \leqq \rho^{-k}} \delta L\left(\left(\frac{x}{\delta}\right)^{1 / k}\right) \\
& =O\left(\sum_{\delta \leq \rho^{-k}} \delta\left(\left(\frac{x}{\delta}\right)^{1 / k}\right)^{k+1 / 2+\epsilon}\right) \\
& =O\left(z^{k+1 / 2+\epsilon} \sum_{\delta \leqq \rho^{-k}} \delta^{-1 / 2 k-\epsilon / k}\right)=O\left(z^{k+1 / 2+\epsilon}\left(\rho^{-k}\right)^{1-1 / 2 k-\epsilon / k}\right) \\
& =O\left(\rho^{-k+1 / 2+\epsilon} z^{k+1 / 2+\epsilon}\right),
\end{aligned}
$$

by Lemma 2.3 .

$$
\begin{aligned}
S_{3}^{\prime} & =\sum_{\delta \leq \rho^{-k}} \delta \sum_{d \leq \rho z} \mu(d) d^{k}=\sum_{\delta \leq \rho^{-k}} \delta L(\rho z)=O\left(\rho^{-2 k}(\rho z)^{k+1 / 2+\epsilon}\right) \\
& =O\left(\rho^{-k+1 / 2+\epsilon} z^{k+1 / 2+\epsilon}\right)
\end{aligned}
$$

by Lemma 2.3 .

Now, substituting the values of $S_{1}^{\prime}, S_{2}^{\prime}$ and $S_{3}^{\prime}$ in $Q_{k}^{\prime}(x)=S_{1}^{\prime}+S_{2}^{\prime}-S_{3}^{\prime}$, we get Lemma 2.5 in virtue of (1.2).

3. Proofs of (1.3) and (1.4). We have by Lemma 2.4 and Lemma 2.5 ,

$$
\Delta_{k}^{\prime}(x)-x \Delta_{k}(x)=O\left(\rho^{k+1} z^{k+1}\right)+O\left(\rho^{-k+1 / 2+\epsilon} z^{k+1 / 2+\epsilon}\right),
$$

for every function $\rho=\rho(x)$ such that $0<\rho<1$. Now, choosing $\rho(x)$ $=x^{-1 / k(4 k+1)}=z^{-1 /(4 k+1)}$, we see that the first $O$-term in (3.1) is $O\left(x^{1+3 /(4 k+1)}\right)$ and the second $O$-term is $O\left(x^{1+3 /(4 k+1)+4 \epsilon /(4 k+1)}\right)$.

Hence (1.3) follows.

We have by partial summation and (1.1),

$$
\begin{aligned}
Q_{k}^{\prime}(x) & =\sum_{n \leq x} q_{k}(n) n=x Q_{k}(x)-\sum_{n \leq x-1} Q_{k}(n)((n+1)-n) \\
& =x Q_{k}(x)-\int_{1}^{x} Q_{k}(t) d t \\
& =\frac{x^{2}}{\zeta(k)}+x \Delta_{k}(x)-\int_{1}^{x}\left(\frac{t}{\zeta(k)}+\Delta_{k}(t)\right) d t \\
& =\frac{x^{2}}{\zeta(k)}+x \Delta_{k}(x)-\frac{x^{2}}{2 \zeta(k)}+O(1)-\int_{1}^{x} \Delta_{k}(t) d t \\
& =\frac{x^{2}}{2 \zeta(k)}+x \Delta_{k}(x)-\int_{1}^{x} \Delta_{k}(t) d t+O(1),
\end{aligned}
$$


so that by (1.2),

$$
\Delta_{k}^{\prime}(x)-x \Delta_{k}(x)=-\int_{1}^{x} \Delta_{k}(t) d t+O(1) .
$$

Hence (1.4) follows in virtue of (1.3). Thus (1.3) and (1.4) are proved.

Finally, we remark that A. M. Vaidya [3] considered the case of square-free numbers and has attempted to give an analytical proof of the stronger result, viz., $\Delta_{2}^{\prime}(x)-x \Delta_{2}(x)=O\left(x^{1+1 / 4+\epsilon}\right)$. But there is a mistake in his proof. He argued on p. 200, lines 16 and 17 of his paper that

$$
\text { “ } O\left(x^{1 / 4+\epsilon} \cdot \lim _{\delta \rightarrow 0}\left(\int_{-T}^{-\delta}+\int_{\delta}^{T}\right)|t|^{3 / 8-k-1+\epsilon}\right)=O\left(x^{1 / 4+\epsilon}\right),
$$

since the integrals converge." The mistake is that the integrals do not converge.

\section{REFERENCES}

1. A. Axer, Über einige Grenzertsatze, S.-B. Akad. Wiss. Wien IIa 120 (1911), 1253-1298.

2. E. C. Titchmarsh, The theory of the Riemann Zeta function, 2nd ed., Clarendon Press, Oxford, 1951. MR 13, 741.

3. A. M. Vaidya, On the order of the error function of the square-free numbers, Proc. Nat. Inst. Sci. India A 32 (1966), 196-201.

4. A. Val'fiš (Walfisz), Weylsche Exponentialsummen in der neueren Zahlentheorie, Mathematische Forschungsberichte, 15, VEB Deutscher Verlag, Berlin, 1963. MR 36 \#3737.

ANDHRA University, Waltair, INDIA 